\title{
Editorial
}

\section{What is Currently Known About the SARS-CoV2 Variants of Concern}

The following four variants of Severe Acute Respiratory Syndrome Coronavirus 2 (SARS-CoV-2) are currently spreading worldwide and are labeled as Variants of Concern (VOC) according to the World Health Organization (WHO): the B.1.1.7 (also called 501Y. V1 or "SARS-CoV-2 VUI-202012/01") variant detected in the United Kingdom (UK) in September 2020, the B.1.351 (also called 501Y.V2) variant discovered in August 2020 in South Africa, the P.1 (also called B.1.1.28.1) variant discovered later in December 2020 in Brazil and Japan, and the B.1.617 variant discovered in India being the most recent addition to the list [1]. The three VOC from UK, South Africa and Brazil can have two mutations in common: the N501Y and E484K spike protein mutations, with the latter being more prevalent in the B.1.351 and P.1 variants [1]. According to the WHO, as of April 20, 2021, the presence of the UK, the South African and the Brazilian variants was reported respectively in 125, 67, and 43 countries, and as of May 2021, the Indian variant was recorded in 44 countries [1]. Moreover, the VOC phylodynamics are continuously updated on animated maps on the Global Initiative on Sharing All Influenza Data website. On top of these VOC, there are several additional variants of interest that are currently being investigated at national and international levels including the P.2 variant (also called B.1.1.28.2) detected in Brazil, and the CAL.20C (also called B.1.427/B.1.429) variant discovered in California [1].

The UK and South African variants seem to spread more easily than the original virus, with an increased transmissibility of up to $70 \%$ and $50 \%$ respectively [2]. The emergence of these VOC in the US has urged the Centers for Disease Control and Prevention to update their guidance on face masking in February 2021. The agency endorsed double masking or fitting a single mask tighter on the face, while the WHO has not issued a statement yet to support this position [3]. No other new precautions have been proposed.

Based on published analyses from Imperial College London, Public Health Scotland, Public Health England, and University of Exeter, the British government stated in February 2021, that being infected with the B.1.1.7 variant is correlated with an increased risk of hospitalization and death compared to the original COVID-19. However, the absolute risk of death per infection remains low [4]. According to the U.K.'s Office for National Statistics, the symptoms described in people infected with the UK variant as well as the incubation period seem to be similar to those of the original virus [5].
The U.S. Food and Drug Administration warned in January 2021, that any molecular testing method for COVID-19 can yield false negative results if a mutation occurred in the virus' genetic sequence detected by the test [6]. However, there are no major concerns regarding the polymerase chain reaction testing which is less affected by genetic variations due to its ability to detect multiple viral genetic sequences. A recent study concluded that the UK and South African variants have minor or no impact on the sensitivity of COVID-19 genetic testing protocols [7].

The UK variant is modestly more resistant to neutralization by sera from individuals who have received the vaccine against SARS-CoV-2 or have recovered from the disease [8]. However, the South African variant was poorly cross-neutralized by convalescent plasma from individuals infected with the original virus, with a 15.1-fold reduced efficacy compared to neutralization of the variant by plasma from individuals infected with this variant [9]. The UK variant maintained in vitro susceptibility to several monoclonal antibodies that are currently available and used against SARS-CoV2, where as the South African and Brazilian variants showed a marked reduction in in vitro susceptibility to bamlanivimab since they possess the E484K mutation. Imdevimab effectively neutralized South African lineage pseudoviruses containing the mutations $\mathrm{N} 501 \mathrm{Y}, \mathrm{K} 417 \mathrm{~N}$, and $\mathrm{E} 484 \mathrm{~K}$, whereas casirivimab, partially neutralized them [10]. In addition, Gilead has announced that remdesivir, the antiviral drug that has been used against the SARS-CoV-2, should remain effective against the VOC from UK [11].

Most of the currently developed vaccines, including Pfizer, Moderna, AstraZeneca, Johnson \& Johnson, and Novavax, target the spike protein of the virus, the location of most of the variants' concerning mutations. It seems unlikely that COVID-19 could mutate to escape $\mathrm{T}$ cell recognition. $\mathrm{T}$ cells stimulated by the current vaccines against SARS-CoV-2 that should be able to protect against these new variants, as $\mathrm{T}$ cells recognize different parts of the virus. Since COVID-19 vaccines create an immune response to the totality of the spike protein, vaccines should theoretically still be efficacious despite some alterations of antigenic sites in the SARS-CoV-2 variants [12]. The UK variant showed a $\sim 2$-fold increase in resistance to vaccinee sera (Pfizer and Moderna), while the South African variant demonstrated an increase in resistance of $\sim 6.5$ to 8.6 -fold, threatening the 
protective effectiveness of current vaccines [13]. The BioNTechPfizer (BNT162b2) vaccine is slightly less effective against the UK and South African variants, but remains effective against the Brazil variant, and it demonstrated a powerful T-cell response against these variants after patients received the two doses [14]. In addition, a study on the highly vaccinated population of Qatar showed that the Pfizer vaccine remained active against the UK and South African variants with respective estimated effectiveness of $89.5 \%$ and $75.0 \%$ [15]. The Moderna vaccine (mRNA-1273) vaccine can offer protection against the UK variant and is believed to be protective against the South African variant [14]. The neutralization of many variants by the Pfizer and Moderna vaccines is largely preserved. However, the variants carrying the mutations K417N/T, E484K, and N501Y (Brazil and South African variants) are significantly more resistant to neutralization, even in fully vaccinated individuals [16]. The E484K mutation has also been noted in the UK variant conferring an estimated sixfold decrease in sensitivity to immune sera from individuals who received the Pfizer vaccine [17]. The AstraZeneca- University of Oxford (AZD1222) vaccine is effective against the UK and Brazil variants but not against the South African variant, which is why South Africa suspended the use of this vaccine after a study doubted its efficacy against the B.1.351 variant [14]. Novavax vaccine achieved $86 \%$ efficacy against the UK variant in a phase III trial, but $49.4 \%$ efficacy against the South African variant in another trial [18]. Johnson \& Johnson vaccine trials resulted in efficacy of $72 \%$ in the US, $66 \%$ in Latin America, and $57 \%$ in South Africa, where the new South African variant is the most prevalent [19]. The SinoVac Vaccine (Coronavac) is effective against the UK and South African variants [14]. The Indian vaccine (Covaxin) is effective against the UK variant [14]. The SinoPharma vaccine (BBIBP-CorV) is effective against the South African variant [14].

Redesigning the vaccines currently being rolled out worldwide might be required. In fact, several COVID-19 vaccine manufacturers such as Moderna and Johnson \& Johnson are looking into updating their vaccines to match spike mutations in the South African variant. Moderna and Pfizer are also exploring the idea of creating booster shots to improve protection against most variants. COVID-19 vaccines might necessitate periodic updates, as is the case for influenza vaccines. Fortunately, even if a new variant escapes current vaccination efforts, most of the available vaccines are relatively easy to repurpose for new variants [20].

\section{CONFLICTS OF INTEREST}

The authors declare they have no conflicts of interest.

\section{AUTHORS' CONTRIBUTION}

TS and JRH contributed equally in designing and writing the manuscript. SSK supervised and edited the manuscript.

\section{REFERENCES}

[1] COVID-19 Weekly Epidemiological Update. '20210420_Weekly_ Epi_Update_36.pdf'. Data as received by WHO from national authorities, as of 18 April 2021, 10 am CET.
[2] Centers for Disease Control and Prevention (CDC). Genomic Surveillance for SARS-CoV-2. Cases, data, and surveillance. 2020. Available from: https://www.cdc.gov/coronavirus/2019-ncov/casesupdates/variant-surveillance.html (accessed February 17, 2021).

[3] Centers for Disease Control and Prevention (CDC). Variants of the Virus. COVID-19 and your health. 2020. Available from: https://www.cdc.gov/coronavirus/2019-ncov/transmission/ variant.html (accessed February 13, 2021).

[4] Horby P, Bell I, Breuer J, Cevik M, Challen R, Davies N, et al. Update note on B.1.1.7 severity. 2021 [Online]. Available from: https://assets.publishing.service.gov.uk/government/uploads/ system/uploads/attachment_data/file/961042/S1095_ NERVTAG_update_note_on_B.1.1.7_severity_20210211.pdf (accessed February 15, 2021).

[5] Coronavirus (COVID-19) Infection Survey: characteristics of people testing positive for COVID-19 in England, 27 January 2021. Office for National Statistics. Available from: https://www.ons.gov.uk/peoplepopulationandcommunity/ healthandsocialcare/conditionsanddiseases/articles/corona viruscovid19infectionsinthecommunityinengland/characteristics ofpeopletestingpositiveforcovid19inengland27january2021 (accessed February 16, 2021).

[6] U.S. Food and Drug Administration. Genetic variants of SARSCoV-2 may lead to false negative results with molecular tests for detection of SARS-CoV-2 - letter to clinical laboratory staff and health care providers. 2021 [Online]. Available from: https://www. fda.gov/medical-devices/letters-health-care-providers/geneticvariants-sars-cov-2-may-lead-false-negative-results-moleculartests-detection-sars-cov-2 (accessed February 16, 2021).

[7] Arena F, Pollini S, Rossolini GM, Margaglione M. Summary of the available molecular methods for detection of SARS-CoV-2 during the ongoing pandemic. Int J Mol Sci 2021;22;1298.

[8] Wang P, Nair MS, Liu L, Iketani S, Luo Y, Guo Y, et al. Antibody resistance of SARS-CoV-2 variants B.1.351 and B.1.1.7. Nature $2021 ; 593 ; 130-5$.

[9] Cele S, Gazy I, Jackson L, Hwa SH, Tegally H, Lustig G, et al. Escape of SARS-CoV-2 501Y.V2 from neutralization by convalescent plasma. Nature 2021;593;142-6.

[10] Moruf A. Fact sheet for health care providers emergency use authorization (EUA) of bamlanivimab and etesevimab. 2021, p. 34. Available from: https://www.covid19.lilly.com/bam-ete/hcp.

[11] Reuschl AK, Thorne LG, Zuliani-Alvarez L, Bouhaddou M, Obernier K, Hiatt J, et al. Host-directed therapies against earlylineage SARS-CoV-2 retain efficacy against B.1.1.7 variant. bioRxiv 2021 [Preprint].

[12] Lauring AS, Hodcroft EB. Genetic variants of SARS-CoV-2what do they mean?. JAMA 2021;325;529-31.

[13] Ho D, Wang P, Liu L, Iketani S, Luo Y, Guo Y, et al. Increased resistance of SARS-CoV-2 variants B.1.351 and B.1.1.7 to antibody neutralization. Res Sq 2021;rs.3.rs-155394 [Preprint].

[14] Hossain MK, Hassanzadeganroudsari M, Apostolopoulos V. The emergence of new strains of SARS-CoV-2. What does it mean for COVID-19 vaccines?. Expert Rev Vaccines 2021;1-4.

[15] Abu-Raddad LJ, Chemaitelly H, Butt AA, National Study Group for COVID-19 Vaccination. Effectiveness of the BNT162b2 Covid-19 vaccine against the B.1.1.7 and B.1.351 variants. N Engl J Med 2021;NEJMc2104974.

[16] Garcia-Beltran WF, Lam EC, St Denis K, Nitido AD, Garcia ZH, Hauser BM, et al. Multiple SARS-CoV-2 variants escape neutralization by vaccine-induced humoral immunity. Cell 2021; 184;2372.e9-83.e9. 
[17] Collier DA, De Marco A, Ferreira IATM, Meng B, Datir RP, Walls AC, et al. Sensitivity of SARS-CoV-2 B.1.1.7 to mRNA vaccine-elicited antibodies. Nature 2021;593;136-41.

[18] Kwok HF. Review of Covid-19 vaccine clinical trials - a puzzle with missing pieces. Int J Biol Sci 2021;17;1461-8.

[19] Johnson \& Johnson. Johnson \& Johnson Announces Single-Shot Janssen COVID-19 Vaccine Candidate Met Primary Endpoints

\section{Tarek Souaid ${ }^{1, \dagger}$, Joya-Rita Hindy ${ }^{2, \dagger}$, Souha S. Kanj ${ }^{2, *}$}

${ }^{1}$ Faculty of Medicine, Université Saint Joseph, Beirut, Lebanon ${ }^{2}$ Division of Infectious Diseases, Department of Internal Medicine, American University of Beirut Medical Center, PO Box 11-0236, Riad El Solh, Beirut 1107 2020, Lebanon

Corresponding author.Email: sk11@aub.edu.lb

${ }^{\top}$ Both authors contributed equally. in Interim Analysis of its Phase 3 ENSEMBLE Trial. Johnson \& Johnson, Content Lab U.S. Available from: https://www.jnj. com/johnson-and-johnson-announces-single-shot-janssencovid-19-vaccine-candidate-met-primary-endpoints-in-interimanalysis-of-its-phase-3-ensemble-trial (accessed February 15, 2021).

[20] Callaway E, Ledford $\mathrm{H}$. How to redesign COVID vaccines so they protect against variants. Nature 2021;590;15-16. 